\title{
Electrode pad suitability for repeated application to the head and neck
}

Keywords: ambulatory monitoring, electrode pads, physiological monitoring

\begin{abstract}
Electrode pads are an integral component of ambulatory monitoring, providing an interface to conduct and record bioelectrical signals. During a previous clinical investigation involving our device for monitoring patients with dizziness, $53 \%$ of individuals experienced temporary redness and inflammation after wearing standard ECG electrode pads on the face for up to thirty days. We postulated that this was caused by a combination of the material composition of the electrode pads and through skin stripping due to their daily replacement. To test this hypothesis, we undertook a further study to determine which combination of electrode pad and replacement regime was most tolerable to the skin. Participants wore three different electrode pads on both sides of their face for thirty days. Electrode pads on the left were replaced daily, and those on the right on alternate days. Participants were instructed to inspect their skin after removal and to not reapply a new electrode pad if they noticed any unexpected changes to the condition of their skin. Electrode pad performance was measured by the duration of wear. The two best performing electrode pads contained a wet gel rather than a solid, adhesive gel, but the advantage of replacing electrode pads on alternate days was negligible. The chloride concentration of the gels was found to be likely factor in determining tolerability. The results suggest that optimal tolerability for ambulatory monitoring on the head and neck is provided by electrode pads containing a wet gel and a low chloride concentration.
\end{abstract}

\section{Introduction}

Physiological monitoring using adhesive sensor pads plays a significant role in many areas of medical diagnosis, particular for monitoring cardiac rhythm. The durability of these pads is tested in situations where ambulatory monitoring is required $[1,2]$. The $\mathrm{CAVA} \AA$ device has been developed to allow long-term monitoring of eye-movements and requires the application of electrode pads to the face [3]. Preliminary work considering the efficacy and acceptability of the CAVA® device has been undertaken in healthy volunteers [3]. During a clinical investigation, in which seventeen participants were required to wear the device on their face for up to thirty days, the device was able to detect short periods of artificially induced nystagmus buried within days of normal eye movement data (sensitivity of $99.1 \%$ and a specificity of $98.6 \%$ ), and over $80 \%$ of participants were compliant in wearing the device for at least $80 \%$ of each day of their trial. However, following 
the daily replacement of the electrode pads to the face, $53 \%$ of individuals experienced temporary redness and inflammation of their skin at the site of contact with the electrode pads.

Skin irritation is a well-documented risk associated with the use electrode pads in other applications, such as Electrocardiography [4]. A number of causes have been postulated by other studies, including irritation or abrasion by materials within the conductive gel or adhesives [5, 6], and allergic reactions resulting from preexisting allergies or sensitisation to substances over time $[5,7,8]$. It is also known that the repeated application and removal of medical adhesives can lead to soreness through skin-stripping [9]. Whatever the specific cause, it is apparent that the skin-contacting materials in electrode pads facilitate skin irritation, and this has led to a significant body of work seeking to develop 'dry' or 'contactless' electrode pads [10, 11]. To understand the cause of the adverse effect reported during our study, with the view to selecting a more appropriate type of electrode pad for use with our device, a small exploratory study was undertaken. This study was important as it would inform electrode pad selection for other applications that involve the application of electrode pads that adhere to the face.

Electrode pads are produced in many different forms using many different combinations of adhesive and conducting compound. The adhesive pad may be made from foam, tape or fabric, and can be impermeable or breathable, and clear or opaque. The conductive compound may exist as a wet gel, or as a solid adhesive gel, and the hydrogel formulation varies in terms of chloride composition (chloride, multi-chloride, potassium chloride) and chloride concentration $(1 \%, 5 \%$ or $9 \%)$.

We designed a small clinical trial to identify whether:

1. The schedule of electrode pad removal was the main cause of skin damage.

2. The electrode pad composition was the main cause of the skin damage.

\section{Methods}

Fig.1 is placed here. 
Ten healthy individuals were recruited from the Norfolk area to participate in this trial. This study was designed and conducted according to the Declaration of Helsinki of 1975, as revised in 1983, and was reviewed by the London - South East Research Ethics Committee. Participants were required to wear six electrode pads on their face for thirty days (Fig. 1 and Table 1). They wore three different electrode pads in total (Table 1); three on both sides of their face. They replaced the electrode pads on the left-hand side on a daily basis, and those on the right-hand side on alternate days. Participants were supplied with enough single-use electrode pads to follow the replacement regime and were required to return all used and unused electrode pads at the end of the trial to demonstrate compliance. All ten participants wore electrode pad A, which was the electrode pad used during our healthy volunteer trial that motivated this latest study. To test a wide range of electrode pad options, five participants wore electrode pads B1 and $\mathrm{C} 1$, and the remaining five wore $\mathrm{B} 2$ and $\mathrm{C} 2$.

\section{Table 1 is placed here.}

Participants were instructed to remove the electrode pads using a medical adhesive remover foam applicator (Appeel Sterile). These applicators reduce the effect of skin stripping by temporarily inhibiting the adhesive properties of the electrode pads. After removing an electrode pad, participants were required to inspect their skin for evidence of any skin issues beyond what might be expected when removing a sticking plaster. Once an issue was encountered under a specific electrode pad, the participant no longer applied an electrode pad to that site. If no skin issues were observed, participants were allocated an hour to wash before reapplying a new electrode pad. Participants were only able to remove all electrode pads on alternate days, and so showering was limited to alternate days. Participants kept a diary of the dates and times that they replaced the electrode pads, and also used the diary to report the condition of the skin under each electrode pad.

\section{Results}

Fig.2 is placed here.

Ten participants took part in the trial. All ten wore electrode pad A, five wore electrode pads B1 and C1, and the remaining five wore electrode pads B2 and C2. Nine participants were still wearing at least one electrode pad at the conclusion of their 30-day trial. One participant reported redness and swelling of their skin beneath all 
electrode pads after three days. This participant was suspected to have experienced an allergic reaction to the electrode pads materials and was subsequently withdrawn from the trial.

The top performing electrode pad (C1) did not produce any skin redness among the five participants who trialled it, but the other four electrode pads all resulted in a problem in at least two participants. The four electrode pads using a breathable backing outperformed the electrode pads using an impermeable foam, and the top two electrode pads contained a wet gel rather than a solid, adhesive gel.

The results showed a subtle advantage to removing the electrode pads every other day compared to daily replacement, although the difference was not statistically significant. In 50\% (4 of 8) cases where removal was delayed for replacement on alternate days, it was only delayed until the replacement regime permitted the observation of the skin beneath the electrode pad.

\section{Discussion}

The results presented here suggest that the primary cause of the skin redness is the material composition of the electrode pads rather than the frequency of replacement. Previous studies have described skin-stripping as a possible mechanism for causing or exacerbating skin issues, either through the mechanical action of removing the electrode pads, or by the intentional removal of the outer layer of skin to improve signal conductivity $[4,5]$. However, the results presented here suggest that skin-stripping was not the primary cause of the skin issues observed. The slight delay for alternate-day compared to daily replacement is largely explained by the regime delaying observation of the skin beneath the electrode pads. Furthermore, the use of the medical adhesive remover to assist with removal of the electrode pads was shown to largely stop the effect of skin-stripping. Therefore, it is difficult to argue that the inconvenience of showering on alternate days is outweighed by the minor advantage of replacing the electrode pads on alternate days.

Other studies have also reported that skin issues can arise due to long-term wear of ECG electrode pads containing a wet gel[ $[4,10,11]$. Both the gel and adhesives have been hypothesised as the primary cause of irritation[6], as well as the conductive pastes sometimes used instead[5, 6]. Patients can also experience an allergic reaction to the materials within the electrodes pads[7, 8], either because of a pre-existing allergy, or through sensitisation to the materials after long-term wear $[5,8]$. It has also been hypothesised that patients with 
allergies to metals, such as silver or nickel, can experience a delayed reaction as metal ions migrate to the surface of the skin, via the electrolyte gel. However, aside from a single allergic reaction occurring during this study, the pattern of skin redness typically observed in this trial was not believed to be the result of an allergic reaction.

In our study, we postulate that the chemical composition of each gel provides a compelling explanation for the issues observed. The top performing electrode pad contained a wet gel, with a chloride concentration of $1 \%$, compared to $9 \%$ for the next best electrode pad. While the adhesive compound also varied between electrode pads, skin redness was usually confined to the skin directly beneath the gel, rather than the adhesive ring. The best performing electrode pad is marketed for neonatal/paediatric applications, and discussions with the manufacturer confirmed that both the chloride concentration and adhesive properties of the electrode pads are the only aspects that determines its suitability for delicate skin (D Verbickas 2019, personal communication, 16 December). Regarding the backing material used by each electrode pad, we believe it may be incidental that the electrode pads with a breathable backing outperformed the electrode pad using an impermeable foam.

In addition to monitoring the condition of their skin, trial participants also reported that the best-performing electrode pad (C1) could become unstuck from the face during sleep or while sweating. Electrode pad C2, which was selected for its comparatively large adhesive surface area, was shown to stick more effectively but caused more skin issues than $\mathrm{C} 1$. Therefore, we suggest that the ideal solution for long-term ambulatory monitoring is to incorporate the gel from $\mathrm{C} 1$ with the adhesive compound and surface area of $\mathrm{C} 2$.

The results show a clear performance ranking among the electrode pads tested. However, to prove a statistically significant difference between each electrode pad, we would have to undertake a trial involving many hundreds of participants. Additionally, this analysis considers the principle materials contained within the electrode pads, however other materials may be present that are not listed on the manufacturers' datasheets. Also, without a complete list of constituent materials for each electrode pad, we cannot discount that the precise ranking shown has been affected by substances other than those listed on the manufacturers' datasheets.

These findings have direct implications for the CAVA ${ }^{\circledR}$ device, which requires the use of electrode pads on the face for up to a month. However, these results also have implications for other areas of continuous ambulatory 
monitoring involving the face, such as brain activity monitoring (Electroencephalography), muscle activity detection (Electromyography), and other applications involving recording of eye-movements (Electrooculography).

Based on the results of this study, we intend to use the ConMed Huggables electrode pads (C1) with the CAVA $®$ device. In the long-term, we hope that electrode pad manufactures may use these findings to develop electrode pads specifically for long-term, ambulatory monitoring on the head and neck.

\section{Acknowledgements}

This work is funded by the Medical Research Council (MRC) as part of MR/P026265/1, but the MRC were not involved in any other aspect of producing this manuscript.

\section{References}

1. Baig MM, GholamHosseini H, Moqeem AA, et al (2017) A Systematic Review of Wearable Patient Monitoring Systems - Current Challenges and Opportunities for Clinical Adoption. J Med Syst 41:115. https://doi.org/10.1007/s10916-017-0760-1

2. Im C, Seo J-M (2016) A review of electrodes for the electrical brain signal recording. Biomed Eng Lett 6:104-112. https://doi.org/10.1007/s13534-016-0235-1

3. Phillips JS, Newman JL, Cox SJ (2019) An investigation into the diagnostic accuracy, reliability, acceptability and safety of a novel device for Continuous Ambulatory Vestibular Assessment (CAVA). Sci Rep 9:10452. https://doi.org/10.1038/s41598-019-46970-7

4. Marozas V, Petrenas A, Daukantas S, Lukosevicius A (2011) A comparison of conductive textile-based and silver/silver chloride gel electrodes in exercise electrocardiogram recordings. J Electrocardiol 44:189_ 194. https://doi.org/10.1016/j.jelectrocard.2010.12.004

5. H. Tam, J. G. Webster (1977) Minimizing Electrode Motion Artifact by Skin Abrasion. IEEE Trans Biomed Eng BME-24:134-139. https://doi.org/10.1109/TBME.1977.326117

6. Hoon Lee J, Min Lee S, Jin Byeon H, et al (2014) CNT/PDMS-based canal-typed ear electrodes for inconspicuous EEG recording. J Neural Eng 11:046014. https://doi.org/10.1088/1741-2560/11/4/046014

7. Rühlemann D, Kügler K, Mydlach B, Frosch PJ (2010) Contact dermatitis to self-adhesive ECG electrodes. Contact Dermatitis 62:314-315. https://doi.org/10.1111/j.1600-0536.2010.01718.x

8. Weber-Muller F, Reichert-Penetrat S, Schmutz J-L, Barbaud A (2004) [Contact dermatitis from polyacrylate in TENS electrode]. Ann Dermatol Venereol 131:478-480. https://doi.org/10.1016/s01519638(04)93644-7

9. Rippon M, White R, Davies P (2007) Skin adhesives and their role in wound dressings. WOUNDS UK $3: 76$ 
10. Baek J-Y, An J-H, Choi J-M, et al (2008) Flexible polymeric dry electrodes for the long-term monitoring of ECG. Sens Actuators Phys 143:423-429. https://doi.org/10.1016/j.sna.2007.11.019

11. N. Gandhi, C. Khe, D. Chung, et al (2011) Properties of Dry and Non-contact Electrodes for Wearable Physiological Sensors. In: 2011 International Conference on Body Sensor Networks. pp 107-112 
Tables

\begin{tabular}{|c|c|c|c|}
\hline Trial ID & Product Name (Manufacturer) & Backing Material & Gel \\
\hline A & Kendall H124SG (Covidien) & $\begin{array}{l}\text { Polyethylene foam } \\
\text { with Polypropylene } \\
\text { film label }\end{array}$ & $\begin{array}{l}\text { Conductive and adhesive } \\
\text { hydrogel }\end{array}$ \\
\hline B1 & WhiteSensor 40123 (Ambu) & Non-woven (Cloth) & $\begin{array}{l}\text { Chloride-based bio- } \\
\text { adhesive gel }\end{array}$ \\
\hline B2 & Cleartrace Diagnostic (ConMed) & Clear perforated tape & $\begin{array}{l}5 \% \text { Potassium chloride } \\
\text { conductive adhesive gel }\end{array}$ \\
\hline $\mathrm{C} 1$ & Huggables (ConMed) & Clear perforated tape & $\begin{array}{l}1 \% \text { Potassium chloride } \\
\text { wet gel }\end{array}$ \\
\hline $\mathrm{C} 2$ & Invisatrace (ConMed) & Clear perforated tape & $\begin{array}{l}\text { 9\% Potassium chloride } \\
\text { wet gel }\end{array}$ \\
\hline
\end{tabular}

Table 1: The material composition of the gel and backing materials for each electrode pad deployed during the trial. Each participant wore three types of electrode pad: A, B1 and C1 or A, B2 and C2. 


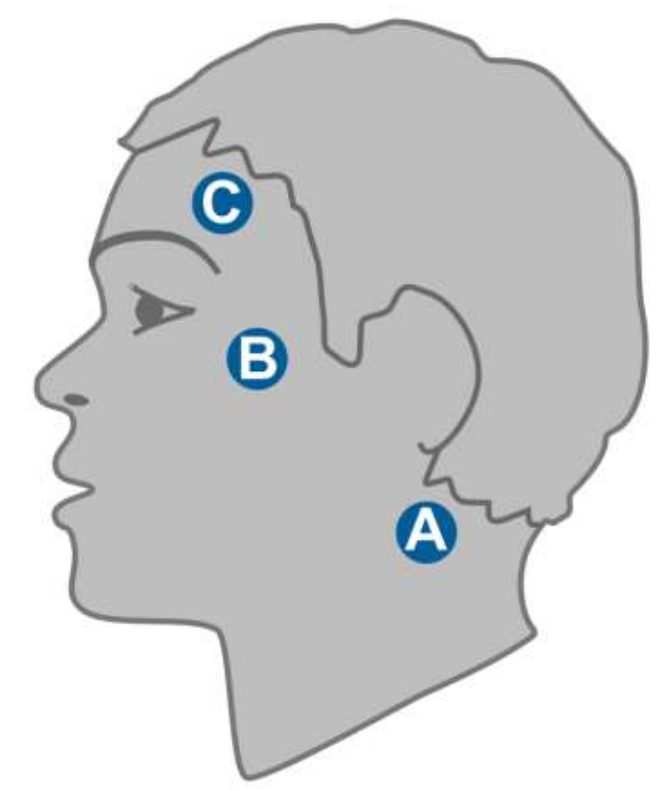

Left-hand side Replace every day

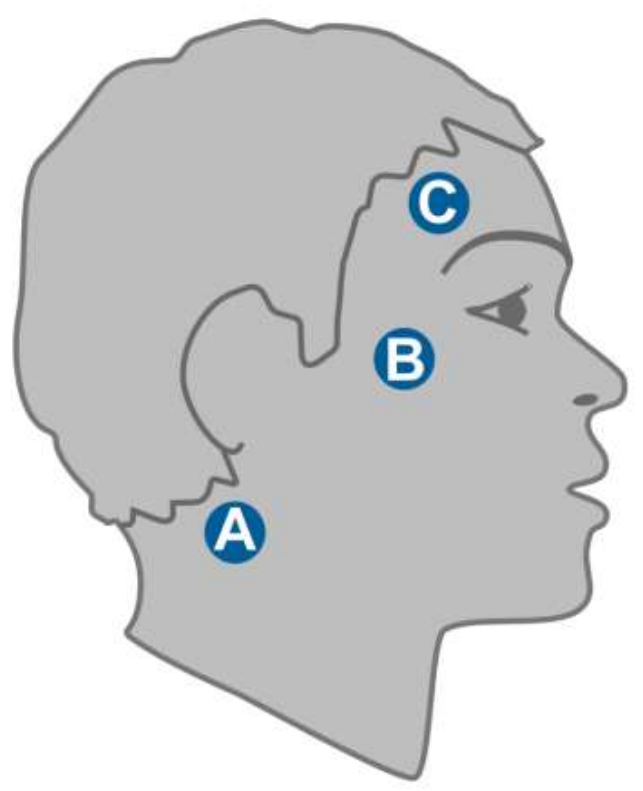

Right-hand side Replace every two days

Fig. 1: A diagram showing the positioning on the face and the frequency of replacement for electrode pads A, B and $\mathrm{C}$. 


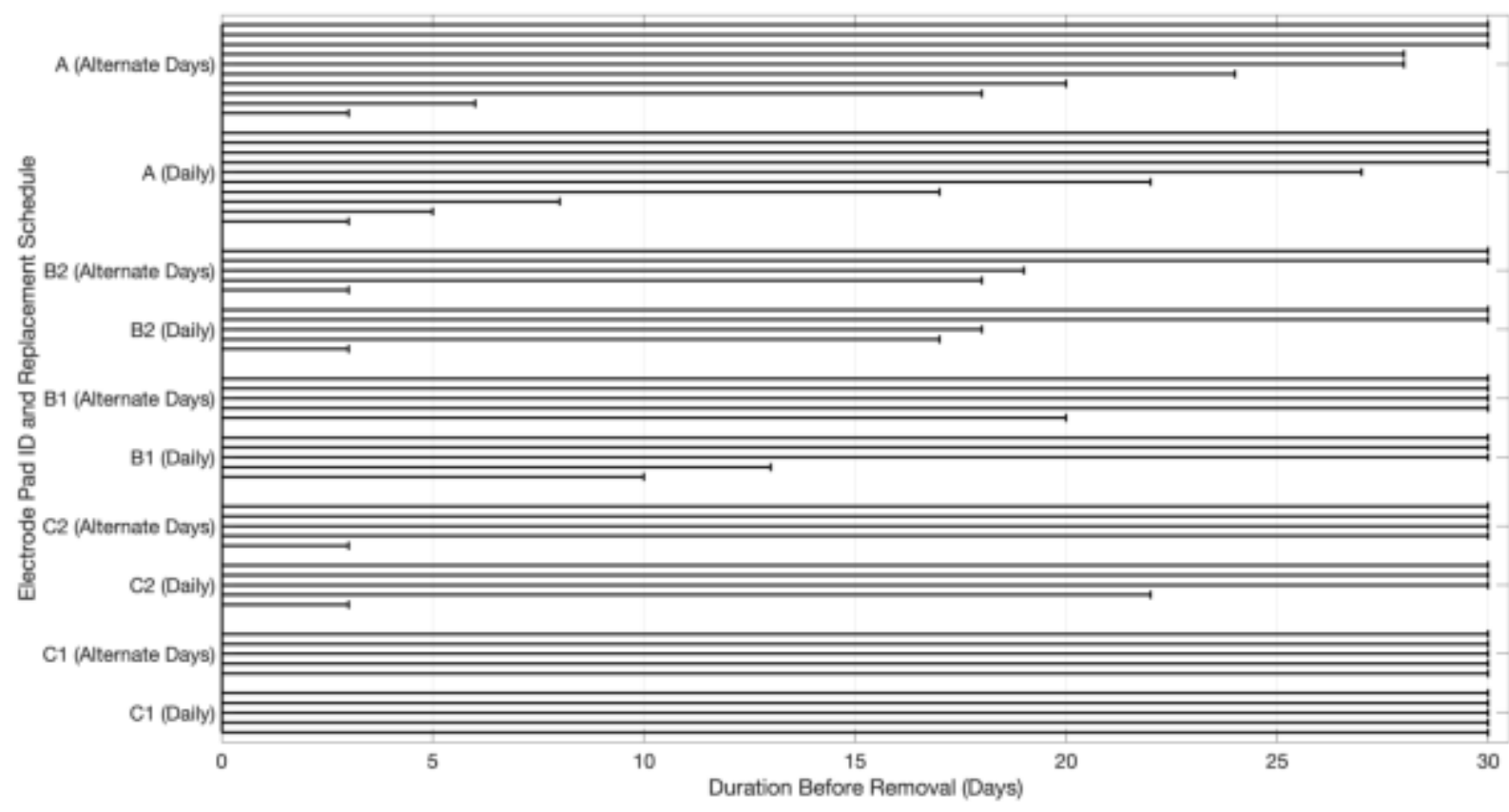

Fig. 2: Plot showing the duration in days that each participant wore each electrode pad. Each participant wore 3 different electrode pads, and five different electrode pads were trialled in total. Electrode pads on the left-hand side of the face were replaced daily and those on the right-hand side on alternate days. 